\title{
The Effect of Flaxseed Extract on Skin Elasticity of The Healing Wound In Rabbits
}

\author{
Abdul Jabbar $O^{a}$, Kashmoola MA', Mustafa Al-Ahmad $B E^{b}$, Mokhtar $K I^{b}$, Muhammad $N^{c}$, Abdul Rahim $R^{d}$, \\ Qouta $L A^{e}$ \\ aDepartment of Oral Maxillofacial Surgery \& Oral Diagnosis, Kulliyyah of Dentistry, International Islamic \\ University Malaysia. \\ ${ }^{\mathrm{b}}$ Fundamental Dental \& Medical Sciences, Kulliyyah of Dentistry, International Islamic University Malaysia. \\ 'Department of Pathology and Laboratory Medicine, Kulliyyah of Medicine, International Islamic University \\ Malaysia. \\ dDepartment of Basic Medical Sciences, Kulliyyah of Medicine, International Islamic University Malaysia. \\ ePhiladelphia University, Jordan, Amman.
}

\section{ABSTRACT}

Introduction: Management of disturbed wounds, large skin defects and the areas where skin tension precludes wound closure is of high clinical importance. Healing in wounds occurs through epithelization and contraction processes (second-intentions healing) that may result in certain undesirable complications including keloid and formation of a fragile epithelial layer. Materials and methods: 27 white New Zealand rabbit included in this study divided into 3 groups; one group of 9 rabbits received Flaxseed gel topically for three time intervals (1, 7, and 14 days); a second group received Fucidin cream as positive control, while a third group has not received any treatment as negative control, Skin elasticity measurements were performed using the DermaLab system. Results: Throughout the study, skin elasticity was significantly greater in Flaxseed group than in others. Flaxseed decrease elasticity value from $(3.46 \pm 2.05)$. Hence, Young's modulus of skin elasticity in flaxseed group was $(2.46 \pm 1.02)$ after 14 days $(p=0.003)$, while no significant differences were evident in both Fucidin group $(1.16 \pm 0.77)$ and non-treated group $(1.86 \pm 1.40)$ $(p=0.019)$, accordingly flaxseed extract more reproducible than other groups demonstrating comparable efficacy in skin elasticity and distensibility. Conclusions: This study showed the therapeutic effect of flaxseed on biologic tissue. Elasticity evaluation demonstrated increased density and firmness in the network of collagen fibers in the dermis and subcutis during wound healing process promise in generating therapeutic gel to be used in wound healing process.

KEYWORDS: Flaxseed, Elasticity, Skin, Wound, Rabbit.

\section{INTRODUCTION}

Using medicinal herbs for curing diseases has been used for a long time. In the past half century, the usage of chemical and synthetic medicines had increased significantly, but their harmful effects on human life has led to a further tendency to

\section{Corresponding Author:}

Asst. Prof. Dr. Basma Ezzat Mustafa Al-Ahmad

Fundamental Dental \& Medical Sciences,

Kulliyyah of Dentistry,

International Islamic University Malaysia,

25200 Kuantan, Pahang, Malaysia.

Tel No : +6095705487

Email : drbasma@iium.edu.my medicinal herbs. ${ }^{1}$ Accordingly medicinal herbs were appreciated and recognised for their aesthetic and ornamental value. To assess the clinical movement of wound scars and to look at results from changing medications and intercessions, it is fundamental to precisely and impartially evaluate the morphological (shading, thickness and flexibility) and physiological attributes (transepidermal water misfortune and water content) of scar tissue. These parameters give data on the development, capacity and nature of the scar. $^{2}$

Elasticity is one of the morphological attributes more as often as possible estimated, despite the fact that there is no accord on the strategy best utilized for assessment of scar versatility in the vertical heading. ${ }^{3}$ 
At present, the most depicted target procedure are suctioned and Transepidermal Water Loss (TEWL), which is thought to be an essential physiological trademark to assess the effectiveness of the skin barrier to work. ${ }^{4}$ The DermaLab (Cortex Innovation, Hadsund, Denmark) can be utilized to assess the two attributes. It consists of two program units, to be specific the flexibility and the TEWL application modes. ${ }^{5}$

Flaxseed is the significant plant-based source of omega-3 fatty acids and recently it has been picking up ubiquity because of its medical advantages. Flaxseed oil is the business type of flaxseed with dynamic use as of late, Its creation presents $57 \%$ of a-linolenic corrosive (ALA, $n-3), 16 \%$ of Linoleic corrosive (LA, n-6), $18 \%$ of polyunsaturated unsaturated fat and $9 \%$ of soaked unsaturated fat. ${ }^{6}$ Flaxseed oil is a profitable supplement for the skin. It has two noteworthy properties: give suitable structure, adaptability and working of cell layers and are fundamental for the blend of intracellular lipids in the stratum corneum. ${ }^{5}$

It has been used for the treatment of wounds, respiratory and gastrointestinal infections, and lymphadenopathy. The compounds of omega-3 polyunsaturated fatty acids have anti-inflammatory properties, which limits inflammation by activating some components of the immune system. ${ }^{6}$

It has been demonstrated that flaxseed supports the immune system, influencing the cells and tissues in charge of invulnerability, for instance, lymph hubs, bone marrow, adrenal organs, and liver. In addition, builds the action of T-lymphocytes and macrophages and quickens wound recuperating. ${ }^{7}$

Thus, this study aimed to evaluate the effectiveness of flaxseed gel in enhancing connective tissue firmness and improving skin texture during wound healing by means of viscoelasticity parameter.

\section{MATERIAL AND METHODS}

\section{Flaxseed oil extraction}

Crude extracted flaxseed oil is freshly prepared from flaxseeds purchased from Jordan. After drying and grinding the plant materials (leaves and stems) are extracted using hexane, Dichloromethane (DCM), and methane as solvents via Soxhlet (continues heat extraction) method, The rotary evaporator (BUCHI Rotavapor R-200) is used to further concentrate the liquid crude extract, and the extract will later on be purified by using Vacuum Liquid Chromatography (VLC) under 800 mbr pressure. ${ }^{8}$

The crude extract used for the in vivo study was dissolved in dimethylsuphoxide (DMSO) $100 \mathrm{mg} / \mathrm{ml}$ and $1 \mathrm{mg} / \mathrm{mL}$ to maintain a stock solution. The final concentration of the extract dilutions will be 100 , $50,25,12.5 \mu \mathrm{g} / \mathrm{ml}$, in a DMSO concentration of $0.01 \%$ to minimize the potential effect of the solvent on cell growth. The oil is then stored shaded from light in a dark place with a temperature of between $15^{\circ} \mathrm{C}$ and $20^{\circ} \mathrm{C}$.

Flaxseed is well-known for the content of chemical compounds with biological activity and functional properties: linoleic, linolenic, oleic, palmitic and stearic acids about 30-40\%. Flaxseed has two major storage proteins, a predominant salt soluble fraction with high molecular weight (11 -12S; globulin; 18.6 $\%$ nitrogen) and a water soluble basic component with low molecular weight (1.6 - 2S; albumin; $17.7 \%$ nitrogen). Polyunsaturated fatty acids (PUFA) omega -3 family, soluble dietary fibers, lignans, vitamins $A$, B, D and E and carbohydrates. ${ }^{9}$

\section{Wounding experimental animals}

Our model of skin wound envelops the actuation and support of numerous procedures which include all skin parts including: epidermis, dermis, hypodermis, veins, and connective tissue. These procedures are evaluated through the investigation of skin properties and the changes in skin cell physiology, including expansion, relocation, separation, framework combination, and tissue rebuilding. At the point when any of these procedures do not work legitimately and the skin's physiological properties are impeded, suitable treatment can avert additionally the skin harm and incite opportune tissue repair.

We composed an in vivo show framework that empowers us to perform uniform, reproducible injury mending tests. Using our philosophy, we have discovered that to achieve measurable significance bunches speaking to each time point or mending parameter ought to incorporate no less than 6 creatures. Stratified randomization of creatures into 
bunches guaranteed even circulation of different qualities, such as gender, weight and age.

The method requires the infliction of a fullthickness skin cut and follow-up of the recuperating time frame on a longitudinal time scale (i.e., 6 creatures for every gathering, for each time point, all injured around the same time). Furthermore, each examination incorporates a quality-control assembles with settled injury recuperating properties (when testing the impact of flaxseed, fusidic corrosive anti-toxin $5 \mathrm{~g}$ cream $2 \%$ regarded amass is utilized as the positive control gathering, while a non-regarded aggregate is utilized as the negative control gathering). This model enabled us to quantitatively analyze the impacts of flaxseed extract with other pharmaceuticals such as Fucidin on the skin's regenerative properties.

Conventional injury infliction methods, which imitate incessant injury mending models incorporate punch and excisional full biopsies, which tend to form nonuniform wounds. ${ }^{10}$ Such injuries deliver nonhomogeneous wound gatherings, which represents a noteworthy issue as far as test reproducibility. In the wake of testing a few surgeries for injuring, we set up a careful strategy that meets our standard criteria. In this system, we present a full thickness $1.5 \mathrm{~cm}$ long incision along the upper back of the rabbit. By 3 hours after wounding, due to skin elasticity and rabbit behaviour, the longitudinal incision point turns into a curved, tear-shaped wounds through all layers of the skin. This specific wounding system produces homogeneous gatherings: it is anything but difficult to perform and is profoundly reproducible. What's more, it guarantees the consistency of the injury length when 3 hours in the wake of injuring just the width shifts.

Experimental rabbits were maintained in good health and just before wounding they were examined and found to be free from any clinical signs of disease. Prior to, and during wounding, rabbits were anesthetized with general anaesthesia using ketamine $(50 \mathrm{mg} / \mathrm{kg})$ and xylazine $(5 \mathrm{mg} /$ $\mathrm{kg}) .{ }^{11}$ Rabbit's eyes were hydrated with normal saline in order to prevent corneal defect due to anaesthesia induced eye dehydration. Rabbits were shaved with electric clippers on their dorsum prior to wounding, then two longitudinal full thickness incisional wounds were made on each experimental animal at the interscapular region, one on either side of midline using a surgical scalpel blade no. 15. Then, an elasticity probe was placed on a predetermined area over the wounded skin and then elasticity was recorded digitally. After recovering from anaesthesia and stabilizing, rabbits were returned to their communal cages.

We followed the wound-healing process over a period of 14 days, with daily application of flaxseed and Fucidin twice daily, examining various healing parameters on days 1,7 , and 14 . The experiments were performed with acute animal-model systems, each comprising of 9 rabbits per group. In order to characterize wound healing, we focused on tissue remodelling and scar formation of the healing process through elasticity measurement.

\section{Evaluation of skin elasticity}

For assessment of skin flexibility changes, the DermaLab USB versatility test (Cortex Innovation ApS, Hadsund, Denmark) was connected over wounded skin areas of all groups. Subsequent to connecting the test to the skin with tape, the interscapular skin of wound area was measured only once to prevent skin fatigue caused by repeated measurements as shown in figure 1.

The DermaLab USB elasticity probe quantifies skin changes and restores forces in accordance with inhalation of skin and the duration of the inhalation, and the results were analyzed using DermaLab USB analysis software, version 1.09 (Cortex Technology ApS). Young's modulus (E) was used for elasticity analysis, which is the value representing the difference in forces to raise surface skin as much as $1.5 \mathrm{~mm}$, the distance between two infrared sensing wires within the probe.

Its unit of measure is the mega pascal (MPa). High $\mathrm{MPa}$ esteems show that a higher vacuum quality was required to lift the skin, which mirrors a more prominent level of skin firmness. Young's modulus $(E)$ decreases with a higher elasticity, indicating that skin elasticity was improved. The estimating precision determined by the producer is $\pm 2 \%$. Estimations were always taken before each therapy session and at the same skin location and marked with a special body marker. 


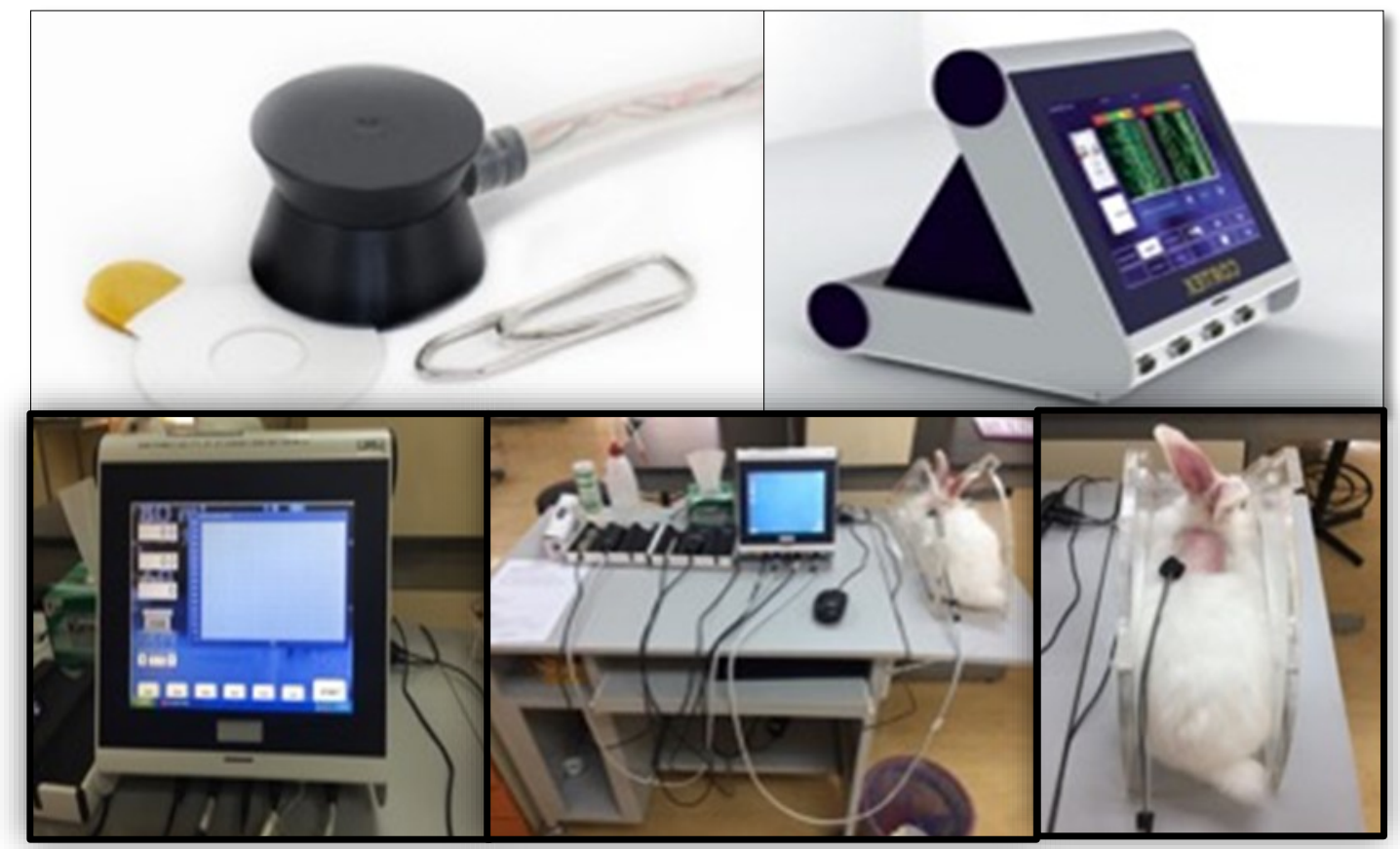

Figure 1: DermaLab skin elasticity suction probe (Cortex Technology, Hadsund, Denmark)

\section{Statistical analysis}

Data is presented as the means \pm S.D. A one-way analysis of variance (ANOVA) was used to compare the Young's modulus of elasticity among the three groups. The research facility information of the three groups were looked at utilizing an examination of covariance (ANCOVA) after an interval of days. All probability values were twotailed. The $\mathrm{p}$ value $<0.05$ was considered statistically significant.

\section{RESULTS}

The characteristics of the mean values of the topical application of a flaxseed extract on hairless rabbit skin for 14 days consecutively are demonstrated in Table 1. However, the Young's modulus of elasticity (E) did not show any significant changes between all treated groups. The mean values were highly decreased in the flaxseed group compared to Fucidin and the normal (nontreated) groups. The skin elasticity results of the flaxseed group measured from the $1^{\text {st }}$ day of the therapy revealed a value of $(3.46 \pm 2.05)$ ( $p<$ 0.071 ), after day $14^{\text {th }}$ of follow-up, skin elasticity of the flaxseed group had improved by $(2.46 \pm 1.02)$ respectively $(\mathrm{p}<0.002)$. In contrast, the improvement in skin properties achieved by Fucidin cream generally ranged between $(1.16 \pm 0.77)$ on the 1st day post treatment and by $(1.00 \pm 0.34)$ on day 14 which was less when compared with flaxseed and non-treated groups.

Table 1: Changes in skin elasticity after applying test materials.

\begin{tabular}{lllll}
\hline & Groups & Mean & $\begin{array}{l}\text { Std. } \\
\text { Deviation }\end{array}$ \\
\hline \multirow{4}{*}{ Day 1 } & No treatment & 2.43 & 0.65 & 9 \\
& Fucidin & 1.16 & 0.77 & 9 \\
& Flaxseed & 3.46 & 2.05 & 9 \\
& No treatment & 1.93 & 0.27 & 9 \\
Day 7 & Fucidin & 1.33 & 0.83 & 9 \\
& Flaxseed & 2.63 & 0.73 & 9 \\
& No treatment & 1.86 & 1.40 & 9 \\
& Fucidin & 1.00 & 0.34 & 9 \\
Day 14 & Flaxseed & 2.46 & 1.02 & 9 \\
\hline
\end{tabular}

A non-significant difference in the mean $(E)$ over time was neither found when considered alone nor when compared within groups. But there was a significance difference when compared between groups as in table 2 . 
Table 2: Mean values of $\mathrm{E}$ over time compared between groups.

Tests of Between-Subjects Effects

Measure: MEASURE_1

Transformed Variable: Average

$\begin{array}{llllll}\text { Source } & \begin{array}{l}\text { Type III } \\ \text { Sum of } \\ \text { Squares }\end{array} \quad \text { df } & \begin{array}{l}\text { Mean } \\ \text { Square }\end{array} & \text { F } & \text { Sig. }\end{array}$

\begin{tabular}{lccccc}
\hline Groups & 47.51 & 1 & 47.51 & 81.85 & .00 \\
Trtn & 2.90 & 2 & 1.45 & 2.50 & .09 \\
Groups * & 31.54 & 2 & 15.77 & 27.17 & .00 \\
Trtn & & & & &
\end{tabular}

Although, the negative control group, which received no prior specific therapy to minimize altered skin firmness from $(2.43 \pm 0.65)$ to $(1.86 \pm$ 1.40 ), thus elasticity vs. time tended to show minor improvement between the studied groups as shown in figure 2.

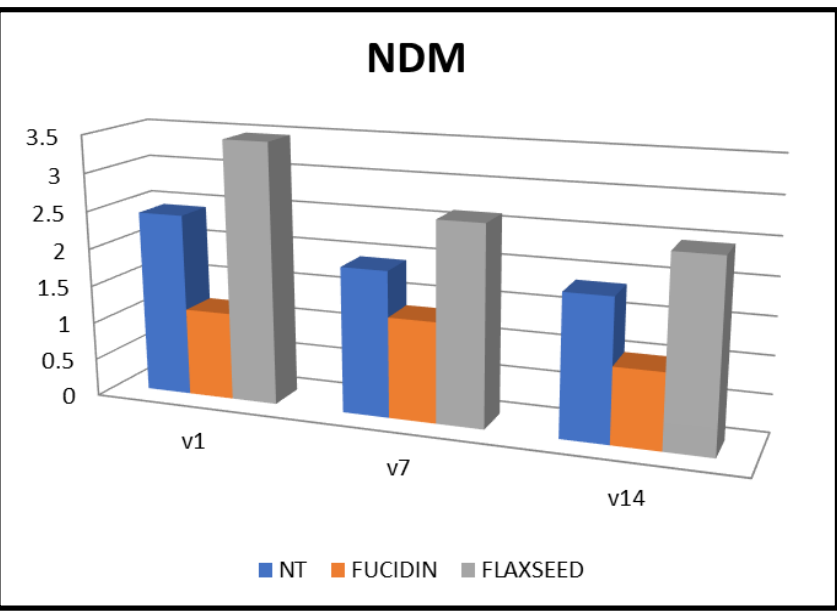

Figure 2: Effects of topical application of flaxseed extract on skin elasticity $(v)$ : all results are expressed as values of measured elasticity on days 1, 7, and 14 among all groups (mean \pm S.D.; $n=9$ ).

In this study, the skin elasticity (E) did not show a significant difference $(p<0.071)$ in each study group, but on the other hand, Greenhouse-Geisser test revealed a significant difference between groups collectively $(0.151)$ as shown in table 2 . As displayed by Dermalab confirming flaxseeds therapeutic use in skin protection. The efficacy is regulated by skin-improving constituents of lignin, alpha linonic acid and alpha linoleic acid that upgrade collagen creation by means of a collagen empowering impact in dermal fibroblasts.

\section{DISCUSSION}

Evaluating therapeutic interventions of extract requires sensitive, solid and substantial estimations. Existing investigations utilizing clinician or patientrated measurements of physical skin properties suggest that these scales have poor dependability, and subsequently legitimacy, that may restrict their incentive as result measures. ${ }^{12}$ Objective measurements potentially offer greater accuracy and sensitivity in quantifying fibrosis. As well, a continuous rather than discrete scale may be more sensitive to change.

In this study, we utilized the DermaLab Suction Cup to evaluate the degree of fibrosis objectively. This device has been investigated in multiple clinical settings such as active burn scars. $^{3}$ age-related connective tissue weakness response following treatments. ${ }^{1}$ vaginal prolapse. ${ }^{13}$ and mapping of normal breast elasticity. ${ }^{14}$

To assess the reproducibility of this apparatus, two independent clinicians measured skin elasticity. The mean difference in the measure of elasticity between clinician $A$ and $B$ appeared to be random. Furthermore, the DermaLab Suction Cup's elasticity measurement yielded a concordance correlation coefficient suggesting a strong level of concordance between the two measurers, for both flaxseeds treated and normal skin tissue. These results thus suggest that the DermaLab generates reproducible results.

Other devices have previously been studied as an objective measurement of skin elasticity with various limitations. Leung et al. first reported using an ultrasound probe and a computer algorithm in $2002 .{ }^{15}$ The deformation of the soft tissue due to a measured applied force was measured. Huang et al. ${ }^{16}$ tested the same system, using a minimized version of the tissue ultrasound palpation system used by Leung et al. ${ }^{15}$ Twenty-seven animals which some received flaxseed and others Fucidin cream were studied. The mean value of flaxseed oil treated wounds reduced generally more than other groups, which are comparable to the DermaLab Suction Cup results. It was noted, however, that the use of this device had some limitations. Stringent measurement of site consistency was required to maintain interand intra-animal test consistency. It was also estimated that several hours of training were 
required for an ultrasonography technician to learn the technique. In contrast, the DermaLab Suction cup does not require precise delineation of the skin site and any healthcare worker may use the device with minimal training.

Most importantly, the elasticity value in the Fucidin treated group had increased slightly (from 1.16 to 1.33). Contrariwise, the flaxseed group showed an increase in its value (from 3.46 to 2.46 ) and the non -treated group (from 2.43 to 1.86 ) in our study for the DermaLab Suction Cup. This was in concordance with all aforementioned studies, ${ }^{3}$ which also demonstrated that objective measurements have better reproducibility than clinical scales. The results for the flaxseed's effect on skin elasticity are summarized in Table 1. However, the use of this device also had its restrictions. The plastic disc, with its diameter of $2 \mathrm{~cm}$, was rather large, therewith limiting an even positioning of the device and thus accurate measurement of tissue compliance. For instance, areas such as the interscapular region and the skin folds which have convex or concave surfaces were not assessable. The DermaLab Suction cup's $2 \mathrm{~cm}$ diameter, in contrast, allowed more versatility with less anatomical limitations. Therefore, implementation in clinical settings will be easier, especially for animals with narrow and non-flat back surfaces.

Davis et al. studied the usefulness of the biomechanical tissue characterization device (BTC2000) in evaluating the parameters of tissue laxity and energy absorption in 41 patients who were treated for soft tissue sarcoma by surgery alone and surgery with radiotherapy. ${ }^{17}$ This method of measuring post-radiation fibrosis was the most similar to the DermaLab Suction Cup, having a laser beam system measuring the skin deflection by sub atmospheric pressure through a chamber aperture, promising quantitative measures of soft-tissue fibrosis were generated.

Correlating our results between the flaxseed and the Fucidin statistically showed a significant association between the groups with a higher elasticity measurement for flaxseed, compared to Fucidin and the non-treated group as shown in table 2. The elasticity also correlated with the flaxseed with a higher value of 3.46 than the non-treated group of 2.43. This suggests that the higher the elasticity measurement value, the less the tissue fibrosis is affected by chronic wound-induced fibrosis. This is consistent with many studies that have demonstrated that decreased fibrosis results in greater improvement in functionality. ${ }^{18}$ The measurements of the suction cup device, thus correlate with both animal skin and natural extract reported measures of functional outcome. This has not been demonstrated with other devices.

Our results suggest that the Dermalab suction cup device generates reproducible elasticity measurements that correlate well with subjective scores as shown in Table 1 . It had been well established that flaxseed associated wounds offered a greater reduction in modulus of elasticity value when compared with Fucidin and non-treated groups. It should also be noted that with the intention to minimize the study's obtrusiveness and to assure high consistence rates, this study was conducted on vital skin tissue and therefore provided more direct evidence for understanding the relationship between the extract and skin parameters after application. ${ }^{19}$

Finally, this study was designed to measure the elasticity in wounded skin tissue. It was provided with a standard time interval of 14 days post wound and consecutive measurements performed from the first day. Statistically a non significant difference in modulus of elasticity vs. time after wound procedure was probably because we included a combined group (spontaneously healed skin wounds) in a follow up period shorter than the period expected to be needed for complete scar maturation. ${ }^{3}$ It should also be noted that some baseline skin parameters were quite variable and it would therefore be beneficial to perform a study on a higher number of subjects to allow a clearer conclusion regarding this parameter. Additionally, application of a natural extract over a longer period would be worth testing as this study was conducted over a two week duration which is a considerably short amount of time to detect extract therapeutic effects on skin, reflecting the rabbit's short skin regeneration cycle. ${ }^{20}$

As was expected, this brief period was not enough to raise the consequences of flaxseed therapeutic effects. This might have induced a non-difference between elasticity measurements, since the elasticity of the skin tissue was probably a reflection of the dermal collagen organization, viscous ground 
substance, subcutis and the elastic fibers. ${ }^{21}$ The values of elasticity would abruptly increase and later improve in the period of scar maturation between the time interval of wound healing. ${ }^{22}$ Considering this, a longer study period would provide valuable insights into dose response relationship. While we were unable to show such a relationship in our study since we used a crude flaxseed extract, such an effect might be observed if the extract were to be done over more skin cycles.

\section{CONCLUSION}

In the present study, the topical application of a flaxseed extract over 14 days of skin wound healing showed an additional effect on skin as it improved elasticity as well as skin firmness, while improvement of elasticity was comparable in other animal groups (Fucidin and normal). There was no significant change of elasticity between the flaxseed and the other group, however, this study clearly showed the therapeutic effect of flaxseed extract on biologic tissue, including incitement of microcirculation and change of fibroblastic cell activity. Elasticity evaluation demonstrated increased density and firmness in the network of collagen/elastic fibers in the dermis and subcutis during the wound healing process, promising in the production of therapeutic gel to be used in the wound healing process.

In general, the DermaLab skin elasticity probe can be utilized as a goal, quick, minimal, and noninvasive strategy for flexibility examination of the skin. In clinical trials we recommend to use a mean of repeated measurements of elasticity to increase the significance changes, possible future clinical research and recommendation should include validity testing of DermaLab elasticity mode, impact of anatomical sites on assessment of elasticity and an imminent longitudinal study for this measurement.

\section{ACKNOWLEDGMENTS}

This study was financially supported by the Fundamental Research Grant Scheme (FRGS) Phase 1/2016 (Project ID: FRGS16-010-0509). All the authors would like to thank International Islamic University Malaysia for this financial assistance.

\section{REFERENCES}

1. Christ, C., Brenke, R., Sattler, G., Siems, W., Novak, P., and Daser,A. Improvement in Skin Elasticity in the Treatment of Cellulite and Connective Tissue Weakness by Means of Extracorporeal Pulse Activation Therapy. Aesthetic Surg. J. 2008;28: 538-544.

2. Vercelli, S., Ferriero, G., Sartorio, F., Stissi, V. \& Franchignoni, F. How to assess postsurgical scars: A review of outcome measures. Disability and Rehabilitation 2009;31: 2055-2063.

3. Anthonissen, M., Daly, D., Fleuws, S., Massage, P., Brussel, MV., Vranckx, EV.,

Measurement of elasticity and transepidermal water loss rate of burn scars with the Dermalab. Burns 2013;39: 420-428.

4. Czaika, V., Alborova A., Richter H., Sterry W., Vergou T., Antoniou C., Lademann J., Koch

S. Comparison of transepidermal water loss and laser scanning microscopy measurements to assess their value in the characterization of cutaneous barrier defects. Skin Pharmacol. Physiol. 2011;25: 39-46.

5. Szyszkowska, B., Łepecka-Klusek, C., Kozłowicz, K., Jazienicka, I. \& Krasowska, D. The influence of selected ingredients of dietary supplements on skin condition. Postepy Dermatologii i Alergologii 2014;31: 174-181.

6. Rossi, R., Pastorelli, G., Cannata, S. \& Corino, C. Recent advances in the use of fatty acids as supplements in pig diets: A review. Animal Feed Science and Technology 2010;162: 1-11.

7. Bignotti, A. Intramammary administration of oxygen/ozone mix in oil vehicle blended platelet concentrate as an unconventional therapy in bovine mastitis. First clinical application. (Università degli Studi di Milano, 2014).

8. Patel, M. B. \& Mishra, S. M. Magnoflorine from Tinospora cordifolia stem inhibits a-glucosidase and is antiglycemic in rats. J. Funct. Foods 2012;4: 79-86.

9. Shim, Y. Y., Gui, B., Arnison, P. G., Wang, Y. \& Reaney, M. J. T. Flaxseed (Linum usitatissimum L.) bioactive compounds and peptide nomenclature: Areview. Trends in Food Science and Technology 2014;38: 5-20.

10. Trostrup, H., Thomsen, K., Calum, H., Hoiby, N. \& Moser, C. Animal models of chronic wound care: the application of biofilms in clinical research. CHRONIC WOUND CARE Manag. Res. 2016;3: 123-132. 
11. Rahman, M. N. A., Sukmasari, S., Doolaanea, A. A. \& Qader, O. A. J. A. Potential oral wound healing of topical application of dental gel prepared from Baccaurea angulata fruit in diabetic rats. J. Pharm. Sci. Res. 2018;10: 167 -174 .

12. Davis, A. M., Dische, S., Gerber, L., Saunders, M., Leung, SF., O Sullivan, B. Measuring postirradiation subcutaneous soft-tissue fibrosis: State-of-the-art and future directions. in Seminars in Radiation Oncology 2003;13: 203-213.

13. Epstein, L. B., Graham, C. A. \& Heit, M. H. Systemic and vaginal biomechanical properties of women with normal vaginal support and pelvic organ prolapse. Am. J. Obstet. Gynecol. 2007;197.

14. Sutradhar, A. \& Miller, M. J. In vivo measurement of breast skin elasticity and breast skin thickness. Ski. Res. Technol. 2013;19.

15. Leung, S. F., Zheng, Y., Choi, C.Y.K., Mak, S.S., Chiu, S.K.W., Zee, B., Mak, A.F.T. Quantitative measurement of post-irradiation neck fibrosis based on the young modulus: Description of a new method and clinical results. Cancer 2002;95: 656-662.

16. Huang, Y. P., Zheng, Y. P., Leung, S. F. \& Choi, A. P. C. High Frequency Ultrasound Assessment of Skin Fibrosis: Clinical Results. Ultrasound Med. Biol. 2007;33.

17. Davis, A. M. AILEEN M. DAVIS, GERRAND, C., GRIFFIN, A., HILL, P.R., PH.D., WUNDER, J. M.D., ABUDU, A., BELL, R.S. Evaluation of clinical utility of BTC-2000 for measuring soft tissue fibrosis. Int. J. Radiat. Oncol. Biol. Phys. 2004;60: 286-294.

18. Greaves, N. S., Iqbal, A.S., Hodgkinson, T., Morris, J., Benatar, B., Baguneid, M. Skin substitute-assisted repair shows reduced dermal fibrosis in acute human wounds validated simultaneously by histology and optical coherence tomography. Wound Repair Regen. 2015;23: 483-494.

19. Crichton, M. L., Donose, B.C., Chen, X., Huang, H., Kendal, M.A.F. The viscoelastic, hyperelastic and scale dependent behaviour of freshly excised individual skin layers. Biomaterials 2011;32: 4670-4681.

20. Chen, C. C., Muarry, P.J., Jiang, T.X., Plikus,M.V., Chang, Y.T., Lee, O.K., Wildeltiz, R.B., Chuong. C.M. Regenerative hair waves in aging mice and extra-follicular modulators follistatin, Dkk1, and Sfrp4. J. Invest.

Dermatol. 2014;134, 2086-2096.

21. Corr, D. T., Gallant-Behm, C. L., Shrive, N. G. \& Hart, D. A. Biomechanical behavior of scar tissue and uninjured skin in a porcine model. Wound Repair Regen. 2009;17: 250-259.

22. JOHN MUTURI KIMANI, B. (UON). Comparative Skin Morphology and Wound Healing in Kenyan African Mole Rat (Tachyoryctes Ibeanus) and Naked Mole Rat ( Heterocephalus Glaber). (University of Nairobi, 2013). 